\title{
THE FORELLI PROBLEM CONCERNING IDEALS IN THE DISK ALGEBRA $A(D)$
}

\author{
RAYMOND MORTINI
}

\begin{abstract}
Let $Z(f)$ be the zero set of a function $f \in A(\mathbf{D})$ and $Z(I)=\bigcap_{f \in I} Z(f)$ the zero set of an ideal $I$ in $A(D)$. It is shown that in the disk algebra $A(\mathbf{D})$ every finitely generated ideal $I$ has the weak Forelli property, i.e. there exists a function $f \in I$ such that $Z(f) \cap T=Z(I) \cap T$, where $T$ is the boundary of the unit circle $\mathbf{D}$. On the other hand, there exists a finitely generated ideal $I$ in $A(D)$ such that $Z(f) \neq Z(I)$ for each choice of $f \in I$. This provides us with a negative answer to a problem of F. Forelli [1].
\end{abstract}

1. The disk algebra $A(\mathbf{D})$ is the Banach algebra of all those continuous functions in the closed unit disk $\overline{\mathbf{D}}$ which are analytic in the open unit disk $\mathbf{D}$, under the usual pointwise algebraic operations and the supremum norm.

Let $Z(f)=\{z \in \overline{\mathbf{D}}: f(z)=0\}$ denote the zero set of a function $f \in A(\mathbf{D})$ and $Z(I)=\bigcap_{f \in I} Z(f)$ the zero set of an ideal $I$ in $A(\mathbf{D})$.

In one of his papers $F$. Forelli [1] posed the problem of classifying those ideals in $A$ (D) which have the property that there exists a function $f \in I$ such that the zero set of $f$ agrees with the zero set of the ideal $I$, i.e. for which ideals $I$ do we have $Z(f)=Z(I)$ for a function $f \in I$ ? Such a property will be referred to as the "Forelli property."

It is known that every closed ideal in $A(D)$ has this property. On the other hand, it remained hitherto unsolved whether each finitely generated ideal in $A(D)$ has the Forelli property (Forelli [1, p. 389]). We now solve this problem. The answer, however, will be negative; a fact that was not expected in view of the results in the ring $H(\mathbf{D})$ of all analytic functions in the unit disk $\mathbf{D}$.

Definition 1. An ideal $I$ in the disk algebra $A(D)$ has the "Forelli property" if there exists a function $f \in I$ such that $Z(f)=Z(I)$.

Proposition 1. There exists a finitely generated ideal in the disk algebra which does not have the Forelli property.

Proof. As generators we take the functions $f_{i}(z)=(1-z) B_{i}(z)(i=1,2)$ of v. Renteln [5, p. 139], where $a_{n}=1-n^{-2}$ are the zeros of the Blaschke product $B_{1}$ and $b_{n}=a_{n}+\varepsilon_{n}, \varepsilon_{n}=n^{-2} \exp \left[-\left(1-a_{n}\right)^{-2}\right]=n^{-2} \exp \left(-n^{4}\right)$, the zeros of the Blaschke product $B_{2}$. Let $I=\left(f_{1}, f_{2}\right)$ denote the corresponding ideal. It is now

Received by the editors October 8, 1984 and, in revised form, December 17, 1984. 1980 Mathematics Subject Classification. Primary 30H05, 30D55, 46J15. 
obvious that $Z(I)=\{1\}$. Hence it is sufficient to show that each function $f \in I$ has infinitely many zeros in $\mathbf{D}$.

Let $f=g_{1} f_{1}+g_{2} f_{2}$ belong to the ideal $I$. By the factorization theorem of F. Riesz, we have $f=B g$, where $B$ is a Blaschke product and $g$ is a function in $A(\mathbf{D})$ which vanishes nowhere in D. Then $B=h_{1} f_{1}+h_{2} f_{2}$, where $h_{1}$ and $h_{2}$ are functions of the Nevanlinna class $N$.

This yields the following estimate (see [5]):

$$
\begin{aligned}
\left|B\left(a_{n}\right)\right| & =\left|h_{2}\left(a_{n}\right)\right|\left|f_{2}\left(a_{n}\right)\right| \\
& \leqslant 2 \exp \left(\frac{C}{1-\left|a_{n}\right|}\right) \frac{\varepsilon_{n}}{1-\left|a_{n}\right|}=2 \exp \left(C n^{2}\right) \exp \left(-n^{4}\right)
\end{aligned}
$$

where $C>0$ is a constant that is independent of $n$.

Thus $\lim _{n \rightarrow \infty}\left|B\left(a_{n}\right)\right|=0$. This proves that $B$ has infinitely many zeros in $\mathbf{D}$, i.e. every member of $I$ vanishes infinitely often in $\mathbf{D}$.

2. Before we proceed, we have to give an auxiliary result of Davie, Gamelin and Garnett.

Let $E$ be an open subset of $T$, the boundary of the unit circle D. We denote by $L_{E}^{\infty}$ the set of all functions in $L^{\infty}$ that are continuous in $E$ and by $H_{E}^{\infty}$ the set of all bounded analytic functions in $\mathbf{D}$ that are continuously extendable to $E$.

Lemma (SeE Garnett [2, P. 399, EX. 15c]). Every function of $L_{E}^{\infty}$ can be uniformly approximated by a quotient of the form

$$
\frac{\sum_{i=1}^{N} c_{i} B_{i}}{B},
$$

where $B_{i}(i=1, \ldots, N)$ and $B$ are Blaschke products in $H_{E}^{\infty}$ and $c_{i} \in \mathbf{C}$.

Our preceding results in $\$ 1$ show that one cannot expect that even a finitely generated ideal $I$ in the disk algebra has the Forelli property, i.e. that $I$ contains a function $f$ such that $Z(f)=Z(I)$. Therefore, we are going to modify the question of Forelli by considering merely the boundary zero sets. This leads us to the following definition.

Definition 2. An ideal $I$ in the disk algebra $A(\mathbf{D})$ has the "weak Forelli property" if there exists a function $f \in I$ such that $Z(f) \cap T=Z(I) \cap T$.

We are now able to prove the main result of this paper.

THEOREM. Every finitely generated ideal in the disk algebra has the weak Forelli property.

Proof. Let $I=\left(f_{1}, \ldots, f_{N}\right)$ be a finitely generated ideal in $A(\mathbf{D})$. Our goal is the construction of a function

$$
f=\sum_{i=1}^{N} H_{i} f_{i} \in I
$$

such that $\left|\sum_{i=1}^{N} H_{i} f_{i}\right| \geqslant C \sum_{i=1}^{N}\left|f_{i}\right|^{2}$ holds on $T$, where $C>0$ is a constant. This immediately yields $Z(f) \cap T \subset Z(I) \cap T$, and hence the assertion, because the inclusion $Z(f) \cap T \supset Z(I) \cap T$ holds trivially for each function $f \in I$. 
Let $g_{i}=f_{i}^{2}, E=T \backslash Z(I)$. The inequality of Cauchy and Schwarz yields the estimate

$$
\left|\sum_{i=1}^{N} \frac{\overline{g_{i}}}{\sum_{n=1}^{N}\left|g_{n}\right|} g_{i}\right| \geqslant \frac{1}{N} \sum_{i=1}^{N}\left|g_{i}\right| \text { on } E \text {. }
$$

Because the sum $\sum_{i=1}^{N}\left|g_{i}\right|$ does not vanish in $E$, the functions $q_{i}=\bar{g}_{i} /\left(\sum_{i=1}^{N}\left|g_{n}\right|\right)$ are in $L_{E}^{\infty}(i=1, \ldots, N)$.

We are now going to approximate the functions $q_{i}$ as in the Lemma. More precisely, there exist functions $h_{i} \in H_{E}^{\infty}$ and Blaschke products $B_{i} \in H_{E}^{\infty}$ such that

$$
\left\|q_{i}-h_{i} / B_{i}\right\|_{\infty} \leqslant 1 / 2 N
$$

where $\|\cdot\|_{\infty}$ is the supremum norm in $L^{\infty}$. Thus we have by (1) and (2) the following estimates on $E$ :

$$
\begin{aligned}
\left|\sum_{i=1}^{N} \frac{h_{i}}{B_{i}} g_{i}\right| & \geqslant\left|\sum_{i=1}^{N} q_{i} g_{i}\right|-\sum_{i=1}^{N}\left|q_{i}-\frac{h_{i}}{B_{i}}\right|\left|g_{i}\right| \\
& \geqslant\left(\frac{1}{N}-\frac{1}{2 N}\right) \sum_{i=1}^{N}\left|g_{i}\right| .
\end{aligned}
$$

To get rid of the denominators on the left side of inequality (3), we multiply with $\left|B_{1}\right| \cdot \cdots \cdot B_{N} \mid=1$. Thus,

$$
\left|\sum_{i=1}^{N}\left(\varphi_{i} h_{i}\right) g_{i}\right| \geqslant \frac{1}{2 N} \sum_{i=1}^{N}\left|g_{i}\right| \text { on } E,
$$

where $\varphi_{i}=\prod_{j=1 ; j \neq i}^{N} B_{j}(i=1, \ldots, N)$.

By construction, the functions $\varphi_{i} h_{i} f_{i}$ belong to $H_{E}^{\infty}$. Because the $f_{i}$ are continuous on $T$ and vanish in $Z(I)$, the functions $H_{i}=\varphi_{i} h_{i} f_{i} \in A(\mathbf{D})(i=1, \ldots, N)$. Thus, $\sum_{i=1}^{N} H_{i} f_{i} \in I$ and (4) implies

$$
\left|\sum_{i=1}^{N} H_{i} f_{i}\right| \geqslant \frac{1}{2 N} \sum_{i=1}^{N}\left|f_{i}\right|^{2} \text { on } T(!)
$$

which was to be proved.

REMARK. Our Theorem does not hold for every given ideal in the disk algebra. There even exist countably generated ideals which do not have the weak Forelliproperty, as the following example shows.

EXAMPLE. Let $\left\{z_{n}\right\}$ be a sequence of different points in $T$ such that $z_{n} \rightarrow 1$ $(n \rightarrow \infty)$ and $Z_{N}=\left\{z_{n}: n \geqslant N\right\} \cup\{1\}$. Because the $Z_{N}$ are closed sets of measure zero, there exist by a theorem of Fatou (Hoffman [4, p. 80]) functions $f_{N} \in A(\mathbf{D})$ such that $Z\left(f_{N}\right)=Z_{N}$.

Let $I=\left(f_{1}, f_{2}, \ldots\right)$ be the ideal generated by the functions $f_{1}, f_{2}, \ldots$ in $A(\mathbf{D})$. It is now obvious that $Z(I) \cap T=\{1\}$. But, on the other hand, each function of the ideal $I$ has the fom $f=\sum_{i=1}^{N} g_{i} f_{i}$ for an integer $N$ and suitable chosen functions $g_{i} \in A(\mathbf{D})$. This implies that $Z_{N} \subset Z(f)$. Thus every function $f \in I$ has infinitely many zeros on $T$. Consequently, there exists no function $f \in I$ such that $Z(f) \cap T$ $=Z(I) \cap T$. 
With regard to the Forelli problem, we are also going to investigate the prime ideals in the disk algebra. Whereas the maximal ideals in $A(\mathbf{D})$ satisfy the Forelli property, there exist, on the other hand, prime ideals which do not have even the weak Forelli property.

Proposition 2. In the disk algebra there exist prime ideals which do not have the weak Forelli property.

Proof. First we define the set $M=\{f \in A(\mathbf{D}): z=1$ is not a cluster point of the zeros of the function $f$ on $T$ \}.

It is obvious that $M$ is closed under multiplication. Let $I=\left(f_{1}, f_{2}, \ldots\right)$ be the ideal of the preceding example. By construction, the ideal $I$ does not intersect $M$. By the Lemma of Zorn there exists an ideal $P \supset I$ that is maximal relative to $P \cap M=$ $\varnothing$. This ideal is now prime (see [3, p. 6]). Moreover, the zero set $Z(P)=\{1\}$.

But for every function $f \in P$, the point $z=1$ is a cluster point of the zeros of $f$ on $T$, because $P \cap M=\varnothing$. Thus there exists no function in $P$ such that $Z(f) \cap T=$ $Z(P) \cap T$.

This paper is a part of the author's doctoral thesis at the University of Karlsruhe (Germany).

\section{REFERENCES}

1. F. Forelli, $A$ note on the ideals in the disk algebra, Proc. Amer. Math. Soc. 84 (1982), 389-392. MR 83c 46041.

2. J. Garnett, Bounded analytic functions, Academic Press, New York, 1981. MR 83g 30037

3. L. Gillman and M. Jerison, Rings of continuous functions, Graduate Texts in Math., No. 43, Springer-Verlag, Berlin and New York, 1976. MR 53 \#11352.

4. K. Hoffman, Banach spaces of analytic functions, Prentice Hall, Englewood Cliffs, N. J., 1962. MR 24 \# A 2844.

5. M. v. Renteln, Every subring $R$ of $N$ with $A(\mathbf{D}) \subset N$ is not adequate, Acta Sci. Math. 39 (1977), 139-140. MR 56 \# 5908.

Mathematisches InStitut I, UNiversität KarLSRUHE, KaRLSRUHe, Germany 